\title{
Ausbildungsreform als Chance für einen Musterwechsel im Verständnis pädagogisch-psychologischen Professionswissens
}

\author{
Christian Kraler $(D)$ Ann-Kathrin Dittrich • Fiona MacKay-Falls
}

Angenommen: 4. Dezember 2017 / Online publiziert: 7. Dezember 2017

(C) Der/die Autor(en) 2017. Dieser Artikel ist eine Open-Access-Publikation.

Zusammenfassung Pädagogisch-psychologische Professionalisierungsprozesse sind ein zentraler Bestandteil der Lehrer/innenausbildung. Sie nehmen gegenwärtig einen zunehmend wichtigeren Stellenwert in der Ausbildungspraxis ein. Im vorliegenden Beitrag wird ein im Rahmen einer umfassenden Curriculum-Reform entwickeltes professionsspezifisches Konzept pädagogisch-psychologischen Wissens diskutiert. Basis für dieses Konzept bilden das Wissensmodell von Mittelstrass, Shulmans Konzeptualisierung von Lehrer/innenwissen sowie jüngste empirische Befunde. Die Darstellung des Konzepts mit Fokus auf Aspekte pädagogisch-psychologischen Wissens erfolgt anhand eines konkreten, im Beitrag prototypisch beschriebenen Curriculums zur Lehrer/innenausbildung (Verbund Lehrer/innenbildung West). Dieses wurde in interinstitutioneller Zusammenarbeit von drei Pädagogischen Hochschulen und zwei Universitäten entwickelt. Ziel des Beitrags ist, damit einen Bogen von grundlegenden wissenschaftstheoretischen Überlegungen hin zu fachlichen Fragen und zur konkreten Umsetzung in die ausbildungswirksame Praxis zu spannen. Eine zentrale Erkenntnis aus dem Erstellungs- und Implementierungsprozess ist, dass die Behandlung weniger aber zentraler Konzepte (fundamentale Ideen), die gut aufeinander abgestimmt, vernetzt und mit spezifisch darauf ausgerichteten Schulpraktika verbunden sind, nachhaltigere Lerneffekte bei Lehramtsstudierenden erzeugt.

Schlüsselwörter Musterwechsel · Pädagogisch-Psychologisches Wissen · Curriculumentwicklung · Bildungswissenschaftliche Grundlagen ·

Curriculumhandbuch 


\title{
Rethinking pedagogical-psychological professional knowledge, opportunities for pattern change within teacher education reform
}

\begin{abstract}
Professionalization is a fundamental and core part of teacher education and practice, with pedagogical-psychological knowledge (PPK) playing a pivotal role in curriculum architecture and subsequent professional practice. This paper discusses a process-oriented understanding of PPK, within the context of widespread curriculum reform in Austria and empirical studies (PISA, ITEL). The theoretical framework presented in this article, uses as a basis, the knowledge concept proposed by Mittelstrass and Shulman's influential theory of teacher knowledge. Based on this theoretical framework, a dichotomous model has been developed that can be applied analytically to PPK in initial teacher education. We attempt to present and reflect the content and curricular specification of PPK within a prototypical curriculum developed and implemented by Verbund Lehrer/innenbildung West. This takes into account and encapsulates the scientific landscape from the abstract theory of science and very specific aspects, including the professional questions of concrete implementation of a curriculum into effective practice. One crucial finding seems to be that focusing on well-adjusted and intertwined fundamental ideas that are connected to school practice/pre-service teacher training have a sustainable effect on teacher education students.
\end{abstract}

Keywords Pattern change $\cdot$ Pedagogical-psychological knowledge $\cdot$ Curriculum development $\cdot$ Course handbook

\section{Einführung}

Ziel dieses Beitrags ist, den Implementierungsprozess pädagogisch-psychologischen Professionswissens (im Folgenden kurz ppP) im Kontext der bildungswissenschaftlichen Ausbildungsanteile des Lehramtsstudiums zu diskutieren. Neben den spezifischen curricularen Inhalten wird hierbei weniger ein Augenmerk auf die vollständige Darstellung der konkreten Implementierung in einem österreichischen Entwicklungsverbund (Verbund LehrerInnenbildung West, Tirol-Vorarlberg-Südtirol) gelegt. Vielmehr werden das Potenzial, Gelingensbedingungen sowie Probleme des geschilderten Prozesses dargestellt. Im Kern geht es hierbei um das moderierte gemeinsame interinstitutionelle Zusammenarbeiten von Expert/innen aus bisher getrennten Ausbildungskulturen (Pädagogische Hochschulen: Pflichtschulausbildung, Universitäten, Höhere Schulen). Die inhaltliche Zusammenarbeit dieser Institutionen birgt das Potenzial eines Musterwechsels im Verständnis pädagogisch-psychologischen Professionswissens (vgl. Abschnitt 2.2, Kraler und Schratz 2012).

Im Rahmen der Reform der Lehrer/innenbildung wurden mit dem Bundesrahmengesetz zur Einführung einer neuen Ausbildung für Pädagoginnen und Pädagogen des österreichischen Parlaments vom 12.06.2013 (vgl. Parlament Republik Österreich 2017) die gesetzlichen Grundlagen für neue Lehramtsstudien geschaffen (vgl. Kraler et al. 2017). Die als BA/MA-Studium neu konzipierte Ausbildung wird für den Sekundarstufenbereich (5.-12./13. Schulstufen: Neue Mittelschule, Polytechni- 
sche Schule, Allgemeinbildende Höhere Schule, Berufsbildende Mittlere und Höhere Schule) von den Pädagogische Hochschulen und Universitäten gemeinsam durchgeführt. In Österreich hat sich damit eine historisch bedeutsame Wende ereignet. Mit diesem Gesetz wurde lehramtsausbildungstechnisch die traditionelle Zweigleisigkeit (Seel 2010, S. 178) erstmals substantiell überwunden (Pädagogischen Hochschulen - Pflichtschulbereich, Universitäten - Ausbildungen für Höhere Schulen). Diese Zusammenlegung ist aus Systementwicklungssicht zu begrüßen (vgl. Dedering 2010). Sie birgt abgesehen von mittelfristig prognostizierten ökonomischen Vorteilen die Chance, dass die beiden bisher getrennten Ausbildungssysteme bzw. Institutionen ihre jeweiligen Stärken in ein gemeinsames Curriculum vor dem Hintergrund eines sich vielfach wandelnden Anforderungsprofils an Lehrkräfte einbringen. Der Übergang von einem good bzw. best practice zu einem next practice Modell im Sinn eines qualitativen Entwicklungssprunges (vgl. Kraler und Schratz 2012) hin zu einer möglichst adäquaten, zukunftsfähigen Ausbildung kann über die Kooperation befördert werden. Inwieweit derartige Kooperationsmodelle vom seit mehreren Jahren zu beobachtenden Trend, Lehramtsausbildung institutionell zu verdichten, zu harmonisieren bzw. basierend auf dem Bologna-Prozess einheitlich an Universitäten zusammenzufassen (vgl. Caena 2014, S. 8 ff) beeinflusst werden, wird im vorliegenden Zusammenhang nicht diskutiert.

In Österreich haben Universitäten und Pädagogische Hochschulen in vier Verbundregionen Curricula entwickelt und neu konzipierte Lehramtsstudien für die Sekundarstufe Allgemeinbildung gemeinsam eingerichtet. Im Verbund Lehrer/ innenbildung West (i.F. kurz VLBW) wurden Kooperationsstrukturen eingerichtet, um auf verschiedenen Ebenen Möglichkeiten der Zusammenarbeit zu etablieren. Konkret kooperieren unter der Leitung der Universität Innsbruck für das Lehramtsstudium Sekundarstufe Allgemeinbildung eine weitere Universität und drei Hochschulen. Diese unterscheiden sich v. a. durch ihre institutionelle Geschichte (Meisterlehre mit hohem Praktikumsanteil vs. Fachlehrer/innenausbildung mit Betonung wissenschaftlich-fachlicher Komponenten), die Kompetenzen des jeweiligen in der Ausbildung eingesetzten Personals sowie die Größe der Institutionen. Diese Faktoren bedingen und formatieren jeweils spezifische Studienkulturen, die sich wiederum nachhaltig auf die Curricula und deren organisatorische Umsetzung auswirken (von stark individualisierten bis zu Massenstudienstrukturen).

Im Rahmen der Neukonzeption der Curricula hatte der VLBW beschlossen, die fachlich-fachdidaktischen und schulpädagogisch-schulpraktischen Komponenten unter Leitung der Universität Innsbruck von Fachgruppen, bestehend jeweils aus Expert/innen der Institutionen, zu entwickeln. Im Folgenden wird primär auf die prozessural-strukturellen wie inhaltlichen Aspekte der Bildungswissenschaften bzw. pädagogisch-psychologischen Inhalte eingegangen. 


\section{Interinstitutionelle Curriculumentwicklung}

\subsection{Fachliche Verortung pädagogisch-psychologischen Wissens}

Die Entwicklung eines neuen Curriculums für die Bildungswissenschaftlichen Grundlagen (BWG) im Lehramtsstudium zur Sekundarstufenausbildung (5.-12./ 13. Schulstufe) erfolgt im Kontext unterschiedlichster Bedingungsgefüge und Prozesse. Plastisch kann von einem geographischen large scale - small scale Kontinuum gesprochen werden, das sich von einem internationalen über einen nationalen, regionalen hin zum lokalen und institutionellen Diskurs spannt. Aufgrund des hohen Vernetzungsgrades und teilweiser Personalunionen der Akteure (vgl. z. B. White 2008, S. $20 \mathrm{ff}$ ) ist dieses Kontinuum jedoch weniger linear top down oder bottom up als vielmehr im Sinn eines Wechselwirkungsmodells aller Beteiligten zu verstehen (vgl. Ritzer 2008, S. A-12 ff), was im vorliegenden Zugang über sechs thematischstrukturellen Bezugspaaren referenziert wird (vgl. Abb. 1).

Die in Abb. 1 dargestellten Zusammenhänge sind zudem als Hinweis auf die Bedeutung derartiger Wechselwirkungen (dargestellt über die Linien) für das ppP im Rahmen der Curriculumerstellung zu lesen. Zum Beispiel über Personalunionen auf unterschiedlichen Ebenen, wenn etwa Forscher/innen Erkenntnisse/Modelle sowohl im Rahmen OECD gesteuerter Forschungsprojekte wie auf institutioneller Ebene propagieren. Damit bekommen diese in der Folge auch für konkrete Curricula eine Referenzbedeutung. So wirken derzeit etwa methodologisch stark quantitativ-psychometrisch orientierte Lehr-Lernparadigmen und die damit einhergehenden Verfahren der psychologischen Diagnostik und Testtheorie auf die inhaltliche Ausgestaltung der Curricula ein.

Internationale Dynamik \& Diskurse

- OECD

- Angloamerikanischer Raum

- Deutschsprachige Wege

Lokale Dynamik

- Entwicklungsverbund West

- Kulturen der Ausbildungsinstitutionen

- Sonderfälle (Geographische Lage, Südtirol
Nationale Dynamik

- Bildungspolitik

- Reformen

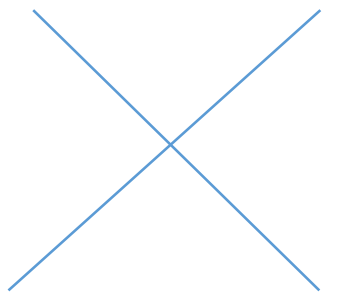

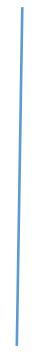

Intrainstitutionelle Entwicklung

- School of Education (Fakultät)

- Fächer, Fachdidaktiken

- Bildungswissenschaften \&

Pädagogisch-Praktische Studien

Abb. 1 Large-small scale Wechselwirkungszusammenhänge zur Lehrer/innenbildung 
Für diese jüngste Entwicklung entscheidend erscheint ein spätestens mit der zweiten Hälfte der 1990er-Jahre eingeleiteter Prozess. Im Gefolge technologischer Möglichkeiten (Internet/Web), einer weiteren Internationalisierung von Bildungsfragen (internationale Schulleistungsvergleichsstudien) sowie damit einhergehender thematisch-disziplinärer Diskussionen ereignete sich eine zweite Empirische Wende in der (Schul-)Pädagogik (vgl. Terhart 2014, S. 12 ff.; bzw. Tippelt und Schmidt 2009, S. 9 ff). Während mit der ersten empirischen Wende die traditionellen Zugänge der geisteswissenschaftlichen Pädagogik mit dem Fokus auf Sinnzusammenhänge um empirische Zugänge erweitert und der Fachbereich vielfach in Erziehungswissenschaften umbenannt wurde (vgl. Jungmann und Huber 2009), erfolgte spätestens mit den Entwicklungen nach 2000 eine weitere terminologische Verschiebung. Die Bildungsforschung prägte aufgrund ihres Aufschwungs in den Jahren nach 2000 die öffentliche Debatte (vgl. Zedler und Döbert 2009, S. 33 f). Dies führte in schulbezogenen Feldern und entsprechend auch in der Lehrer/innenbildung dazu, vom Begriff Schulpädagogik vielfach zum Terminus Bildungswissenschaften überzugehen. In Österreich spricht der Gesetzgeber bei den Rahmenvorgaben für Lehramtsstudien explizit von allgemeinen bildungswissenschaftlichen Grundlagen (BGB1. I Nr. 124/2013). Entscheidend für diese zweite empirische Wende ist die zunehmende Bedeutung von Fragen der Folgenabschätzung und prognostischer Aspekte im Kontext der Bewertung von Bildungssystemen. Die variablenbasierte Konstruktion und quantitativ zahlenmäßige Beschreibbarkeit kausaler und funktionaler Zusammenhänge gewinnt im schulpädagogischen und bildungspolitischen Diskurs ein hohes Maß an Bedeutung. In diesem Kontext wurde und wird pädagogisch-psychologisches Wissen und insbesondere Wissen zur pädagogischen Diagnostik quasi kanonisch einem quantitativen Paradigma zugeordnet. Den aktuellen Studierendengenerationen wird damit in der Folge ein Bild der quantitativen Mess- und Erfassbarkeit pädagogischen Wissens vermittelt. Ob das der Komplexität dieses Wissens in seiner Anwendung bzw. Umsetzung in der unterrichtlichen Praxis gerecht wird, ist zumindest zu hinterfragen.

\subsection{Terminologisch-wissenschaftstheoretischer Zugang}

Zur Frage der Professionalisierung von Lehrkräften liegen inzwischen auch im deutschen Sprachraum reichhaltige Konzepte, Modellierungen und Befunde vor (vgl. Zlatkin-Troitschanskaia et al. 2009; Helsper 2011). Vogel (2016) diskutiert die Generierung und Struktur erziehungswissenschaftlichen Wissens, was für ppP trotz inzwischen erheblich erweiterter empirischer Befundlagen zumindest in der österreichischen scientific community der Lehrerbildungsforschung über weite Strecken noch auszustehen scheint.

Mit den Erziehungswissenschaften als primäre Bezugsdisziplin der schulpädagogisch-bildungswissenschaftlichen Lehrer/innenbildung(sforschung) ist deren Diagnose eines fehlenden integrierenden inhaltlichen Kerns einer sowohl empirischprognostischen als auch normativ orientierten Erziehungswissenschaft (Vogel 2016, S. 452) auch für den entsprechenden Bereich der Lehrer/innenbildung von Bedeutung. Vogel ortet eine Besonderheit der Erziehungswissenschaften in ihren binären Schemata, wobei disziplinäres Wissen/Professionswissen, Theorie/Praxis und For- 
Tab. 1 Curriculare Abbildung des wissenschaftstheoretischen Diskurses zur zweiten empirischen Wende

\begin{tabular}{|c|c|c|c|}
\hline Disziplinärer Dual & Curriculare Benennung & Wissensformen & Bemerkungen \\
\hline Erziehungswissenschaftliche & Bildungswissenschaftliche & Wissenschaftliches & ,interdisziplinär“ mit \\
\hline Inhalte & Grundlagen (BWG) & $\begin{array}{l}\text { Wissen } \\
\text { (Orientierungswissen) }\end{array}$ & $\begin{array}{l}\text { Schulpädagogik als } \\
\text { Teil der EZW als pri- } \\
\text { märer Bezugsdisziplin }\end{array}$ \\
\hline Pädagogik & $\begin{array}{l}\text { Pädagogisch-Praktische } \\
\text { Studien (PPS) }\end{array}$ & $\begin{array}{l}\text { Reflektiertes Hand- } \\
\text { lungswissen der } \\
\text { Praxis } \\
\text { (Verfügungswissen) }\end{array}$ & $\begin{array}{l}\text { PPS umfassen Schul- } \\
\text { praktika und institutio- } \\
\text { nelle Begleitveranstal- } \\
\text { tungen }\end{array}$ \\
\hline
\end{tabular}

schung/Praxisanleitung genannt werden; Bereiche, die für die bildungswissenschaftlichen Anteile in Lehramtsstudien sowohl in der täglichen Auseinandersetzung als auch im Sinn fundamentaler bzw. paradigmatischer Konzepte von zentraler Bedeutung sind. Mit Stichweh spezifiziert Vogel disziplinäres Wissen als zentrales Merkmal zur identitätsstiftenden Charakterisierung der Disziplin (Vogel 2016, S. 545).

Das von Terhart (2014) wie Vogel (2016, S. 456f.) diskutierte Spannungsfeld Pädagogik-Erziehungswissenschaften lässt sich in der inhaltlichen Zuordnung wie curricularen Bezeichnung (letzteres basierend auf den gesetzlichen Vorgaben) produktiv nutzen. In Tab. 1 werden die genannten Spannungsfelder auf ihre curriculare Operationalisierbarkeit in Lehramtsstudien hin strukturell zugeordnet.

Dieser in Tab. 1 dargestellte Zugang wurde im Rahmen der Curriculumentwicklung im VLBW weiter ausdifferenziert, wobei zwei im Folgenden dargestellte Konzepte, ein strukturelles und ein inhaltliches, miteinander verschränkt wurden.

Das inhaltliche Konzept baut auf der gegenwärtig wohl in der scientific community akzeptierten Standardtaxonomie auf, die etwa Hohenstein et al. (2017) wie folgt zusammenfassen: „Professionswissen wird üblicherweise in die Kategorien Fachwissen (Content Knowledge, CK), fachdidaktisches Wissen (Pedagogical Content Knowledge, PCK) sowie pädagogisches bzw. pädagogisch-psychologisches Wissen (Pedagogical Knowledge, PK bzw. Pedagogical/Psychological Knowledge, PPK [...]) der Lehrkraft eingeteilt.“ (Hohenstein et al. 2017, S. 91)

Diese Differenzierung nach Inhalten, Hohenstein et al. (wie bereits Shulman 1986) definieren PPK als schulpädagogisches und pädagogisch-psychologisches Wissen (2017, S. 91 f), lieferte als disziplinterminologisches Sammelbecken einen gemeinsamen Nenner für die unterschiedlichen Kulturen (praxisausgerichtete $\mathrm{Hu}-$ manwissenschaften der Pädagogischen Hochschulen, universitäre forschungsorientierte Schulpädagogik). Um mehr begriffsanalytische Trennschärfe sowohl in der fachlichen Diskussion als auch curricularen Zuordnung zu erreichen, wurde dieser Zugang mit einem strukturellen Konzept verschränkt.

Dieses kann in einem ersten Schritt über eine mengentheoretische Struktur verdeutlicht werden: Mengen- wie definitionstheoretisch wird hierbei von der folgenden Teilmengenrelationen ausgegangen: $\mathrm{K} \supset \mathrm{pK} \supset$ pKiTE. Hierbei ist mit K das ganze Wissen bezeichnet, $\mathrm{pK}$ steht für das pädagogisch-psychologische Wissen und pKiTE für ebendieses Wissen in der Lehrer/innenbildung. Letzteres, basierend auf der englischen Terminologie, ist in diesem Beitrag mit ppP gleichzusetzen. 
Abb. 2 Dimensionen pädagogisch-psychologischen Wissens

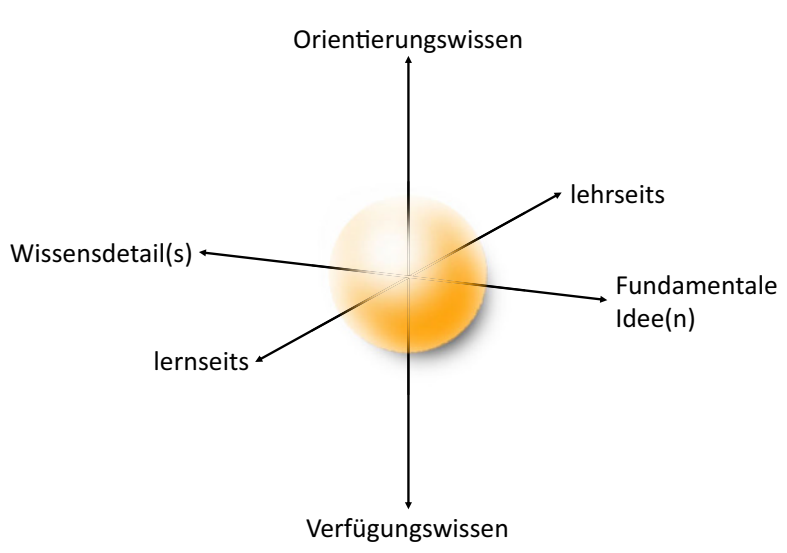

Im zweiten Schritt wurde kontrastierend zur Konzeption in Tab. 1 das dort bereits implizit angedeutete Modell zur Wissensklassifikation von Mittelstraß (2011) verwendet. Dieser teilt Wissen in zwei Formen ein: in Verfügungs- und Orientierungswissen. Verfügungswissen ist ihm zufolge ein Wissen, ,das die Verfügungsgewalt des Menschen über seine Welt vergrößert, begründete, [...]. Verfügungswissen ist ein positives Wissen, ein Wissen um Ursachen, Wirkungen und Mittel [...]. Es beantwortet Fragen nach dem, was wir tun können, aber nicht Fragen nach dem, was wir tun sollen.“ (Mittelstraß 2011, S. 19) Orientierungswissen ,,ist ein regulatives Wissen, ein Wissen um Ziele und Maximen“ (Mittelstraß 2011, S. 19). Letzteres ist ein handlungsorientierendes Wissen. „Ohne die Wahrnehmung dieser Aufgabe entstehen Orientierungsdefizite, wird das Können, das sich im Verfügungswissen zur Geltung bringt, orientierungslos“ (Mittelstraß 2011, S. 19). Mittelstraß eröffnet mit dieser Charakterisierung von Wissensformen in der Lehrer/innenbildung die Möglichkeit, Wissen jenseits der traditionellen Theorie-Praxis Dichotomie (Vogel 2016, S. 454) als handlungsorientiert und handlungsorientierend-regulativ zu fassen. Beide Wissensformen sind sowohl in Lehrveranstaltungen als auch in schulpraktischen Studienanteilen wirkmächtig bzw. relevant, was die professionsspezifische Reflexion in Theorie wie Praxis befördern kann.

Im dritten Schritt werden die beiden Konzepte miteinander verbunden. Pädagogisch-psychologisches Wissen hat sowohl eine Orientierungs- wie eine Verfügungsdimension, die analytisch und damit auch hinsichtlich curricularer Aspekte getrennt werden können.

Unter Berücksichtigung zweier weiterer Dimensionen wurde das in der folgenden Abb. 2 dargestellte Modell pädagogisch-psychologischen Wissens im Rahmen der Lehramtsausbildung im VLBW als Basis für die detaillierte curriculare Planung verwendet.

Wir positionieren sowohl modellierte (vgl. z. B. Hohenstein et al. 2017) wie normativ bzw. präskriptiv generierte pädagogisch-psychologische Wissensbereiche (vgl. Kunter et al. 2011, S. 194) zur Orientierung in diesem dreidimensionalen Modell. Die zweite Dimension definiert den Grad der inhaltlichen Detailliertheit, von fundamentalen Ideen pädagogisch-psychologischen Wissens bis zu Details, basierend auf Bruners (1960) heute in der naturwissenschaftlichen Didaktik (Mathematik/Physik/ 
Informatik) rezipiertem Konzept. Im laufenden Weiterentwicklungs- und Qualitätssicherungsprozess der Arbeitsgruppe BWG wird derzeit versucht, professionsspezifische fundamentale Ideen zu den bildungswissenschaftlichen bzw. pädagogisch-psychologischen Inhalten zu charakterisieren. Diese werden in Anlehnung an Schwill (1993) und Bruner (1960) kriterial definiert (Horizontalkriterium, Vertikalkriterium, Zeitkriterium, Sinnkriterium).

Die dritte Dimension bezieht sich auf das zentrale Kerngeschäft des Unterrichtens. Pädagogisch-psychologisches Wissen entfaltet sich im Berufsalltag im strukturell vom didaktischen Dreieck (Gruschka 2002, S. 87 ff) geprägten Handeln. Bei der Konzeption des Curriculums gehen wir davon aus, dass im Gefolge des bekannten paradigmatic shift from teaching to learning (Wildt 2007, S. 115) ppP auch die didaktische Herausforderung einer Personalisierung des Unterrichts im Kontext des unterrichtlichen Gruppenprozesses antizipieren muss. Schratz et al. (2011) haben hierfür das Begriffspaar lehrseits/lernseits ausgearbeitet. Es bezieht sich auf den didaktischen Musterwechsel im Hinblick auf die generative Dichotomie formaler Bildung, das Wissen (Lehrende wissen und lehren, Schüler/innen lernen). Daraus wird der pädagogische Auftrag des Lehrens im Sinn eines Konzeptions- und handlungsleitenden Musterwechsels abgeleitet, Unterricht vom Lernen her zu denken, zu konzeptionieren und zu planen.

\section{Struktureller Implementierungsprozess pädagogisch-psychologischen Wissens im Curriculum}

Ende 2013 konstituierte sich eine interinstitutionelle bildungswissenschaftliche Arbeitsgruppe zur Erstellung eines gemeinsamen Curriculums für alle Studienfächer der Sekundarstufe Allgemeinbildung. Die Herausforderungen für dieses Studium waren vielfältig. Zentral hierbei war und ist, dass es sich nicht um eine einfache Studienplanänderung oder Entwicklung für ein bestehendes Studium handelt, auch wenn es auf bestehenden Angeboten aufbauen mag. Vielmehr war eine grundsätzlich neue Architektur zu entwickeln, da es dieses gemeinsame Lehramt in Österreich zuvor nicht gab. Parallel hierzu waren curricular neueste Reformentwicklungen des österreichischen Schulwesens zu berücksichtigen (Neue Mittelschule, Reform der gymnasialen Oberstufe, standardisierte Reifeprüfung, individualisierte Unterstützungsangebote in den Schulformen usw.). Nicht zuletzt erfolgte mit dieser Studienplanarchitektur der Übergang vom Diplom- zum BA/MA-Konzept. Die vielfältigen damit verbundenen Fragen waren systemisch mit vielen institutionellen und personellen Beharrungstendenzen (Angst vor Neuem, Verlust von Einflussnahme und Steuerungsrechten, Machtverlust) von Aktanten im System verbunden (vgl. zum grundsätzlichen Phänomen: Zymek 2004, S. 216).

Organisations- und curriculumsentwicklungstechnisch war daher die zentrale Herausforderung, bereits im Planungsstadium einen echten strukturellen wie inhaltlichen Musterwechsel zu befördern. Schratz (2008) hat allgemeine Gelingensbedingungen für einen solchen Musterwechsel konzipiert. Ziel des konkreten Prozesses war, nicht aus vorhandenen institutionellen Konzepten einen Kompromiss oder im günstigen Fall ein best practice Modell abzuleiten (,,mehr vom Gleichen“). Die qualitätsent- 
Abb. 3 Lehrveranstaltungsund modulübergreifende thematische Gliederung pädagogischpsychologischen Wissens über die Ausbildungszeit

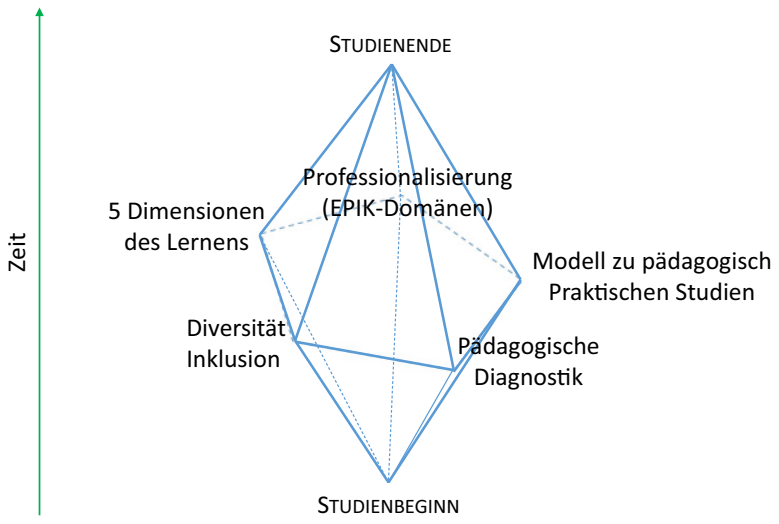

wicklungstechnische Kernfrage war vielmehr, welche Art von „Störung oder Intervention“ einen Musterwechsel im Denken und Konzeptionieren innerhalb der Expert/innengruppe befördern kann, um zu einem next practice Modell zu kommen. Ausgangsprämisse war, dass die innovative Umsetzung einer gemeinsamen Ausbildung aller Sekundarstufenlehrer/innen am besten über eine Zusammenarbeit aller beteiligten Expert/innen realisiert werden kann. Um im gemeinsamen Prozess das prospektive Moment dynamisch zu befördern, wurde mit einer Zielvision gearbeitet (vgl. Schratz 2007, S. 35 ff), der Frage nach einer Lehrer/innenbildung für die Schule 2030. Hierbei bekam die Expert/innengruppe zum Einstieg ein Set von Fragen für einen Lernort Schule 2030 mit.

Zur Umsetzung arbeitete ein Expertengremium von etwa 35 Personen über eineinhalb Jahre in einer Arbeitsgruppe „Bildungswissenschaftliche Grundlagen und Pädagogisch Praktische Studien“ zusammen. Moderiert und strukturell geplant wurde dieser Prozess vom Institut für LehrerInnenbildung und Schulforschung der Universität Innsbruck als gesetzlich letztverantwortlicher Institution für die Umsetzung des Curriculums. Zur Entwicklung der bildungswissenschaftlichen Inhalte wurde eine top-down Vorgangsweise in der Moderation eingebracht und in ein fünfstufiges Vorgehen übersetzt, um darüber die Gesamtkomplexität der Inhalte (vgl. Abschnitt 2.2.) bearbeitbar zu machen:

1. zentrale bildungswissenschaftliche Themenfelder basierend auf dem wissenschaftlichen state of the art und der gelebten Lehrer/innenbildungspraxis möglichst exakt benennen,

2. daraus eine fachlogisch kohärente Grobstruktur für Modul- und Lehrveranstaltungstitel und deren Abfolge im Curriculum ableiten,

3. Erstellung und Ausformulierung des Studienplans/Curriculums, basierend auf den gesetzlichen und institutionellen Vorgaben (Kompetenzorientierung, Definition von Lernergebnissen usw.),

4. inhaltliche Detailplanung der Module und Lehrveranstaltungen inkl. didaktischer Umsetzungsmodelle,

5. basierend darauf, Erstellung eines Curriculumhandbuchs 
Die Expert/innen der beteiligten Institutionen arbeiteten im Rahmen von sechs ganztägigen Workshops mehrheitlich in thematischen Subarbeitsgruppen (vgl. Abb. 3), die je durch eine integrierende Plenarstunde abgeschlossen wurden. Die jeweiligen Ergebnisse wurden in einseitigen Curriculum-Briefs festgehalten.

Das zentrale materialisierte Ergebnis der gemeinsamen Arbeit war ein 140-seitiges Curriculumhandbuch. Dieses beschreibt in einem einleitenden Vorwort grundlegende und alle Module durchlaufende durchgängige architektonische, didaktische und inhaltliche Prinzipien. Leitende didaktische Prinzipien hierbei waren bzw. sind:

- Backward Design (vgl. Wiggins und McTighe 2005),

- Spiralprinzip (Bruner 1960),

- exemplarisch-prototypisch-genetischer Zugang (Wagenschein 1968),

- Bildungsgangprinzip (Professionsbiographie, Subjektiver-Objektiver Bildungsgang) $\rightarrow$ Personalisierung \& Professionalisierung (Kraler und Schratz 2012),

Wesentlich war, dass die genannten didaktischen und inhaltlichen Aspekte für die komplexen Curriculuminhalte eine orientierende (Orientierungswissen, vgl. Abschn. 2.1.) und kohärenzstiftende Funktion haben.

Ein weiteres Moment ergab sich am Ende des Prozesses in der Rückschau auf die Gesamtarchitektur hinsichtlich der Frage nach thematischen roten Fäden durch das Gesamtcurriculum im Sinn orientierender Querstrukturen. Hierbei waren nicht nur Überlegungen hinsichtlich der Professionalisierung der Studierenden leitend, sondern auch die Frage, wie Lehrerbildner/innen aus den unterschiedlichen Institutionen sowie künftigen neuen Kolleg/innen über das Curriculumhandbuch hinaus der Blick aufs Ganze vermittelt werden kann. In Abb. 3 ist die im interinstitutionellen Prozess von den Expert/innen gemeinsam entwickelte inhaltliche Struktur dargestellt.

Die fünf in Abb. 3 benannten Bereiche zeigen die inhaltliche Schwerpunktsetzung der pädagogisch-psychologischen Ausbildung im VLBW auf. Zu Studienbeginn sollen diese Bereiche über die einzelnen Inhalte und die Studienzeit entfaltet werden, um sich zu Studienende zu einem ersten kohärent-integrativen Wissensbestand zu entwickeln. Insgesamt entstanden durch den Prozess ein hohes Maß interinstitutionellen Commitments und eine gemeinsame terminologische Basis hinsichtlich der Verwendung von Konzepten und Begriffen für die Lehrveranstaltungen im Studienangebot.

\section{Konkrete Inhalte pädagogisch-psychologischen Wissens im BA-Curriculum}

Basierend auf dem Wissensmodell (Abb. 2) sowie der inhaltlichen Aufgliederung (Abb. 3) wurden die pädagogisch-psychologischen Felder über den bildungswissenschaftlichen Teil im Lehramtsstudienplan wie in Tab. 2 dargestellt im BA-Studienverlauf verankert.

Im ersten Modul wird sowohl theoretisch in das bildungswissenschaftliche big picture überblicksmäßig-orientierend (Orientierungsfunktion) als auch praxisorientiert im Sinn eines self-assessments eingeführt. Vorlesungen vermitteln hierbei das Wissen in seiner Systematik (Orientierungswissen). In Proseminaren (Teilungszif- 
Tab. 2 BA-Curriculum pädagogisch-psychologische Grundlagen

\begin{tabular}{|c|c|c|c|c|}
\hline Semester & Titel & SSt & ECTS & $\begin{array}{l}\text { Davon } \\
\text { päd.-prakt. } \\
\text { Studien in } \\
\text { ECTS-AP }\end{array}$ \\
\hline
\end{tabular}

1. Pflichtmodul: Professionsspezifische Initiierung im Berufsfeld Schule

1. Semester

VO Schule als Bildungsinstitution \& Rolle 2

2

0

der Lehrperson

PS Proseminar zu ,Schule als Bildungsinsti- 2 tution und Rolle der Lehrperson "

2. Semester

PR Umgang mit professionsspezifischen

Herausforderungen des Berufsfelds Schule

2

2

und Schulpraktikum 1

Teilsumme

6

7,5

2. Pflichtmodul: Lernen, Lehren und Forschen im Diversitätskontext

3. Semester

VO Lernen und Lehren im Diversitätskontext

PS Lernen und Lehren im Diversitätskontext

VO Bildungsforschung und Entwicklung von

Schule und Unterricht

PS Bildungsforschung und Entwicklung von

Schule und Unterricht

Teilsumme

2

20

121

$1 \quad 1,5 \quad 0$

$1 \quad 2 \quad 1$

$\mathbf{7 , 5}$

3. Pflichtmodul: Diagnostizieren, Beraten, Erziehen, Unterrichten und Beurteilen

4. Semester

VO Diagnostik und Beratung

2

PS Diagnostik \& Beratung

22

5. Semester

PR Erziehung \& Leistungsbeurteilung und
Schulpraktikum 2

2

Teilsumme

6

7,5

4. Pflichtmodul: Professionsspezifische Profilierung als Lehrperson im Berufsfeld Schule

6. Semester

PS Professionsspezifisches Wissen und Han-

2

3

3 deln

7. Semester

PR Professionsspezifisches Wissen und Han-

2

$2+5$

7 deln und Schulpraktikum $3 a+3 b$
Teilsumme
4
10
10

5. Pflichtmodul: Integration und Vertiefung professionsspezifischer Kompetenzen \& Wahlfächer

8. Semester PS Integration professionsspezifischer Kom-
petenzen

\section{Teilsumme}

2

6. Pflichtmodul: Aktuelle Themen zur Schul- und Bildungsforschung

Abwechselnde LV zu aktuellen bildungswis- $\quad 2$ senschaftlichen Themen bzw. Vertiefungen zu Themen der Module 1-4

VO Gendersensible Aspekte/Lebensweltliche Mehrsprachigkeit und Interkulturalität/ Inklusion und Heterogenität im Schul- und Bildungssystem

\section{Teilsumme}

Summe

2

27
$2,5 \quad 2$

$2,5 \quad 2$

2,5

0

$4 \quad 5 \quad 0,5$




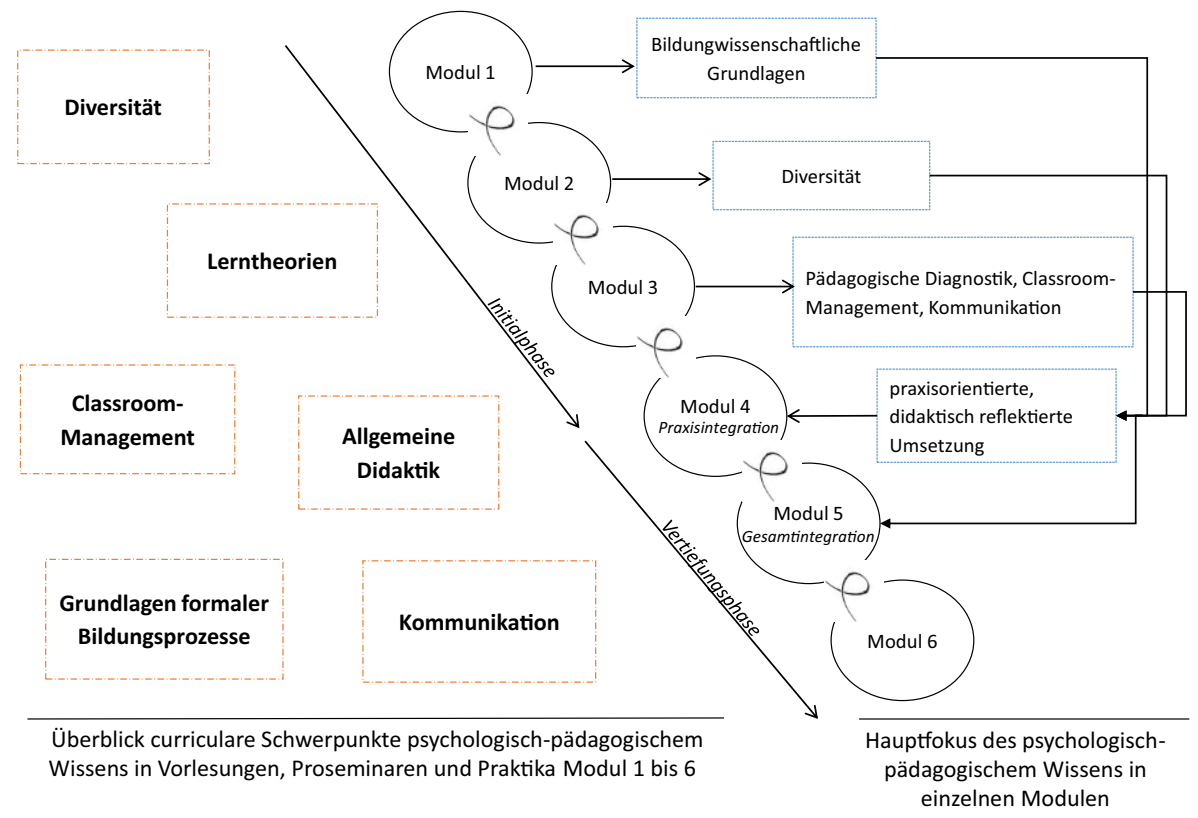

Abb. 4 Zentrale Elemente pädagogisch-psychologischen Wissens im BA-Lehramtsstudium

fer 20) und Praktika soll dieses Wissen in ein erstes Probehandeln transformieren werden (Verfügungswissen). Modul 2 vertieft darauf aufbauend das konzeptionelle und theoriebasierte Verständnis für Lehrprozesse unter besonderer Berücksichtigung von Fragen der Heterogenität und Diversität verbunden mit einer pädagogisch-psychologischen bzw. bildungswissenschaftlichen Wissenschaftspropädeutik. Basierend auf grundlegendem Wissen um das Bildungssystem und vertieftes Wissen um Lernen und wissenschaftliche Methoden werden in Modul 3 zentrale diagnostische Felder aus pädagogischer Perspektive behandelt: Diagnostik, Beratung, Erziehung und Leistungsbeurteilung, verbunden mit einem Schulpraktikum mit Fokus auf diese Aspekte. Im Pflichtmodul 4 werden die erworbenen Kompetenzen in umfangreichen Schulpraktika erprobt. Während in Modul 1 in den Praktika neben systematisch beobachtendem Hospitieren vorrangig kurze eigenständige Sequenzen unterrichtet werden sollen, in Modul 3 der Fokus auf diagnostischen Elementen liegt, zielt Modul 4 auf fundierten eigenständigen Unterricht ab. Die Studierenden haben zu diesem Zeitpunkt ihr fachliches, fachdidaktisches und pädagogisch-psychologisches Grundwissen erworben. In Modul 5 werden diese Erfahrungen basierend auf den Elementen aus Abb. 4 integriert. Die Bedeutung einer solchen Lehrveranstaltung stützt auf Erfahrungen aus dem auslaufenden Sekundarstufencurriculum der Universität Innsbruck. Es zeigt sich durchgängig, dass Lehramtsausbildungen nur wenig Orte zur Integration der gesamten erworbenen Kompetenzen bieten. Frühere eigene Untersuchungen haben nahegelegt, einen ersten explizit gewidmeten Ort am Ende des BA-Studiums zu platzieren. Die Wahlfächer (Modul 6) schließlich können ab dem zweiten Studiensemester besucht werden. 
Mit der folgenden groben Analyse soll ein Überblick gegeben werden, welches konkrete ppP im VLBW vermittelt werden soll und wo es im oben erwähnten Curriculumhandbuch in den einzelnen Modulen verankert ist. Eine Clusterung unter Berücksichtigung der curricular-modularen Verortung der Themen ergibt das folgende in Abb. 4 dargestellte Bild.

Die einzelnen Schwerpunkte werden ab dem Modul 4 über punktuelle Momente hinaus verstärkt vertiefend aufgegriffen und vernetzt thematisiert (Spiralcurriculum). Modul 6 nimmt als Wahlfachmodul eine Sonderstellung ein. Im Vergleich zu den bisherigen Curricula der Pädagogischen Hochschulen und Universitäten im Verbund zeigt sich, dass pädagogisch-psychologischem Grundlagenwissen im BA-Studium ein größeres Gewicht gegeben wird. Der besondere Fokus liegt bei den Themen Diversität, Heterogenität, Diagnostik, Lerntheorien, evidenzbasierte didaktische Konzepte zum Lernen und Lehren, Kommunikation sowie Klassenführung.

Insgesamt spiegeln sich die curricularen Anforderungen an Lernergebnisse im Curriculumhandbuch in den folgenden zu erwerbenden Aspekten besonders wider:

- Strategien professioneller metakognitiver Reflexion entwickeln (insbesondere studierendenseitige Antizipation und handlungstransformatorische individualisierte Aneignung professionsspezifischer pädagogisch-psychologischer Inhalte und Prozesse),

- Orientierungs- in Handlungswissen transformieren durch die Schulpraktika,

- Sensibilisierung für Lernseitigkeit,

- Entwicklung eines Professionsbewusstseins,

- die Ausbildung als Startpunkt lebenslangen professionellen Lernens verstehen.

Als eine bereits hier vorweggenommene zentrale Erkenntnis aus dem Erstellungsund inzwischen auch Implementierungsprozess ergab sich, dass die Behandlung weniger aber zentraler Konzepte (fundamentale Ideen), die gut aufeinander abgestimmt, vernetzt und mit spezifisch darauf ausgerichteten Schulpraktika verbunden sind, nachhaltigere Lerneffekte erzeugen. Zumindest zeigt sich ein solches Bild bei Analyse der Klausuren zu den Vorlesungen, die von allen Studierenden absolviert werde müssen.

\section{Resümee}

Ziel dieses Beitrags war, den Prozess der Erstellung eines Curriculums für pädagogisch-psychologische Anteile im Lehramtsstudium (Sekundarstufe) an einem Beispiel prototypisch und reduziert auf zentrale Faktoren zu beschreiben. Pädagogisch-psychologische Studienanteile (im Curriculum als bildungswissenschaftliche Grundlagen bezeichnet) entfalten im Sinn Latours „Parlament der Dinge“ (Latour 2010) eine Eigendynamik, die durch Schulreformen und die damit verbundene Notwendigkeit, insbesondere pädagogisch-diagnostische Inhalte umfassender bereits in die Ausbildung einzubringen, verstärkt wird. Die multifaktoriellen inhaltlichen, verwaltungstechnischen und legistischen Reformprozesse im Bildungssystem bedingen institutionelle Entwicklungsprozesse, die zu einem fundamentalen Wandel im Ausbildungsverständnis führen. Die hohe Veränderungsdynamik im System ist zudem 
auf personaler und institutioneller Ebene teilweise mit Angst, Abwehr und anfänglichen Blockadebestrebungen verbunden.

Der systembedingte Aufbruch bietet und birgt jedoch für pädagogisch-psychologische Anteile einer Lehramtsausbildung ein großes Entwicklungspotenzial. Die quantitative Aufwertung dieser Studienanteile alleine läuft institutionell gebunden Gefahr, zu einem Mehr vom Gleichen zu führen. Die vom Gesetzgeber vorgesehene Zusammenarbeit der verschiedenen Institutionen bringt erstmals Expert/innen mit unterschiedlichen beruflichen Sozialisationen zusammen, die nach einer inhaltlichen und gruppendynamischen Verständigungsphase gemeinsam ein neues Curriculum erstellen können. Als entscheidende Gelingensbedingungen stellten sich hierbei folgende Faktoren heraus: klare Moderationsstrukturen, handhabbare Instrumente zur Sicherung des jeweiligen Ergebnisstandes und zur Kommunikation zwischen Subarbeitsgruppen, die diskursive Einigung auf fundamentale, detailübergreifende thematische Querinhalte (fundamentale Ideen), konkrete Kollaboration beim Formulieren und die Verschränkung inhaltlicher mit (hochschul-) didaktischen Konzepten, das handhabbare Zurverfügungstellen aktueller Befunde und ausgewählter Forschungsliteratur. Der inhaltliche Musterwechsel konnte auf organisationaler wie personeller Ebene realisiert werden, indem sich alle Akteure in ihren Anliegen und Expertisen wahrgenommen und verstanden fühlten.

Als zentral für den im Beitrag diskutierten Prozess erwiesen sich zwei Aspekte. Erstens eine zugrundeliegende theoretische Konzeption pädagogisch-psychologischen Wissens (Abb. 2), die sich basierend auf einem thematischen Gliederungsmodell (Abb. 3) in ein Curriculum um- und übersetzen setzen lässt. Und zweitens das hochschuldidaktische Instrument des Curriculumhandbuchs. Dieses ist als ein im Detail dynamisches, in seiner Grundarchitektur jedoch beständiges Qualitätsentwicklungsinstrument sowie Belegstück mit Diskursfunktion in der Auseinandersetzung mit den Fachdidaktiken/Fächern zu verstehen. Die didaktische und inhaltliche Ausformulierung im Handbuch liefert einen differenzierteren Einblick in die konkrete Lehrveranstaltungsarchitektur als das allgemeiner und abstrakter formulierte Curriculum. Die Notwendigkeit orientierender inhaltlicher roter Fäden durch das gesamte Studium sowie die Präzisierung von Schwerpunktsetzungen werden befördert, da es ein konkretes Produkt gibt, an dem man pädagogisch-psychologische Studieninhalte in ihrer curricularen Wechselwirkungsdynamik übersichtlich und handhabbar darstellen kann.

Funding Open access funding provided by University of Innsbruck and Medical University of Innsbruck.

Open Access Dieser Artikel wird unter der Creative Commons Namensnennung 4.0 International Lizenz (http://creativecommons.org/licenses/by/4.0/deed.de) veröffentlicht, welche die Nutzung, Vervielfältigung, Bearbeitung, Verbreitung und Wiedergabe in jeglichem Medium und Format erlaubt, sofern Sie den/die ursprünglichen Autor(en) und die Quelle ordnungsgemäß nennen, einen Link zur Creative Commons Lizenz beifügen und angeben, ob Änderungen vorgenommen wurden. 


\section{Literatur}

Bruner, J. (1960). The process of education. Cambridge Mass: HUP.

Bundesrahmengesetz zur Einführung einer neuen Ausbildung für Pädagoginnen und Pädagogen, BGBl. I Nr. 124/2013.

Caena, F. (2014). Initial teacher education in Europe: an overview of policy issues. ET2020 Working Group on Schools Policy. Brussels: EUROPEAN COMMISSION.

Dedering, K. (2010). Entscheidungsfindung in Bildungspolitik und Bildungsverwaltung. In H. Altrichter \& K. Maag Merki (Hrsg.), Handbuch Neue Steuerung im Schulsystem (S. 63-80). Wiesbaden: VS.

Gruschka, A. (2002). Didaktik. Das Kreuz mit der Vermittlung. Wetzlar: Büchse der Pandora.

Helsper, W. (2011). Lehrerprofessionalität - der strukturtheoretische Professionsansatz zum Lehrberuf. In E. Terhart \& al (Hrsg.), Handbuch der Forschung zum Lehrerberuf (S. 149-170). Münster: Waxmann.

Hohenstein, F., Kleickman, T., Zimmermann, F., Köller, O., \& Möller, J. (2017). Erfassung von pädgogischem und psychologischem Wissen in der Lehramtsausbildung. Entwicklung eines Messinstruments. Zeitschrift für Pädagogik, 1/2017, 91-113.

Jungmann, W., \& Huber, K. (Hrsg.). (2009). Heinrich Roth - „moderne“ Pädagogik als Wissenschaft. Weinheim: Juventa.

Kraler, C., \& Schratz, M. (2012). From best practice to next practice: a shift through research-based teacher education. Reflecting Education, 8(2), 88-125.

Kraler, C., Fügenschuh, B., \& Reich, K. (2017). PädagogInnenbildung Neu im Verbund LehrerInnenbildung West. Eine Standortbestimmung zu Gelingensbedingungen und Herausforderungen. Zeitschrift für Hochschulrecht, 16, 79-86.

Kunter, M., Baumert, J., Blum, W., Klusman, U., Krauss, S., \& Neubrand, M. (Hrsg.). (2011). Professionelle Kompetenzen von Lehrkräften. Ergebnisse des Forschungsprogramms COACTIV. Münster: Waxmann.

Latour, B. (2010). Das Parlament der Dinge: Für eine politische Ökologie. Frankfurt a.M.: Suhrkamp.

Mittelstraß, J. (2011). Glanz und Elend der Geisteswissenschaften. Grünwald: Komplett-Media.

Parlament Republik Österreich (2017). Bundesrahmengesetz zur Einführung einer neuen Ausbildung für Pädagoginnen und Pädagogen. https://www.parlament.gv.at/PAKT/VHG/XXIV/I/I_02348/index. shtml;. Zugegriffen: 9. Apr. 2017.

Ritzer, G. (2008). Sociological theory. Boston: McGraw-Hill.

Schratz (2007). Qualität sichern. Schulprogramme entwickeln. Seelze: Kallmeyer.

Schratz, M. (2008). Von best practice zu next practice. Musterwechsel braucht Leadership. In U. StadlerAltmann, J. Schindele \& A. Schraut (Hrsg.), Neue Lernkultur - neue Leistungskultur (S. 331-344). Bad Heilbrunn: Klinkhardt.

Schratz, M., Schwarz, J., \& Westfall-Greiter, T. (2011). Auf dem Weg zu einer Theorie lernseits von Unterricht. In W. Meseth, M. Proske \& F. Radtke (Hrsg.), Unterrichtstheorien in Forschung und Lehre (S. 103-115). Klinkhardt: Bad Heilbronn.

Schwill, A. (1993). Zentralblatt für Didaktik der Mathematik. Fundamentale Ideen der Informatik, 1, 20-31.

Seel, H. (2010). Einführung in die Schulgeschichte Österreichs. Innsbruck: Studienverlag.

Shulman, L.S. (1986). "Those who understand: knowledge growth in teaching". Educational Researcher, 15(2), 4-14.

Terhart, E. (2014). Erziehungswissenschaften und Lehrerbildung. Münster: Waxmann.

Tippelt, D., \& Schmidt, B. (Hrsg.). (2009). Handbuch Bildungsforschung. Wiesbaden: VS.

Vogel, P. (2016). Die Erziehungswissenschaft und ihr Wissen. Selbstkritik, Thematisierungsformen, Analytik. Zeitschrift für Pädagogik, 7-8/2016, 452-473.

Wagenschein, M. (1968). Verstehen lehren. Genetisch - Sokratisch - Exemplarisch. Weinheim, Basel: Beltz.

White, H. (2008). Identity \& control. How social formations emerge. Pronceton, Oxford: PUP.

Wiggins, G., \& McTighe, J. (2005). Understanding by design. 2nd expanded edition. Alexandria: ASCD.

Wildt, J. (2007). On the way from teaching to learning via competences as learning outcomes. In A. Pausits \& A. Pellert (Hrsg.), Higher education management and development in central, southern and Eastern Europe (S. 115-124). Münster: Waxmann.

Zedler, P., \& Döbert, H. (2009). Erziehungswissenschaftliche Bildungsforschung. In R. Tippelt \& B. Schmidt (Hrsg.), Handbuch Bildungsforschung (S. 23-45). Wiesbaden: VS.

Zlatkin-Troitschanskaia, O., Beck, K., Sembill, D., Nickolaus, R., \& Mulder, R. (Hrsg.). (2009). Lehrerprofessionalität. Bedingungen, Genese, Wirkungen und ihre Messung. Weinheim: Beltz.

Zymek, B. (2004). Geschichte des Schulwesens und des Lehrberufs. In W. Helsper \& J. Böhme (Hrsg.), Handbuch der Schulforschung (S. 205-240). Wiesbaden: VS. 